\section{Chrischta Ganz}

\section{Die sieben Planetenmetalle}

Mit dem Ausdruck «die sieben Planetenmetalle» werden bezeichnet:

- Gold, das Metall der Sonne,

- Silber, das Metall des Mondes,

- Quecksilber, das Metall des Merkurs,

- Kupfer, das Metall der Venus,

- Eisen, das Metall des Mars,

- Zinn, das Metall des Jupiters, und

- Blei, das Metall des Saturns.

Auch wenn sowohl Sonne als auch Mond aus naturwissenschaftlicher Sicht nicht zu den Planeten zählen, so wurden sie von Paracelsus (14931541), von Rudolf Steiner (1861-1925), von Alla Selawry (1913-1992) und von vielen anderen Heilkundigen zur kosmischen Siebenheit gezählt [1]. Paracelsus lehrte über die Planetenkräfte [1]:

«Die sieben Planeten haben in keinem Ding eine grössere Wirkung als in ihren Metallen, die sie mit all ihren Eigenschaften und Kräften durchdringen, als wenn sie selbst mit ihrem ganzen Wesen da wären. So wirkt die Sonne vornehmlich im Golde, der Mond im Silber, Merkur im Quecksilber, Venus im Kupfer, Mars im Eisen, Jupiter im Zinn und Saturn im Blei.»

\title{
Metalle in der Naturheilkunde
}

Diese Art der Welterfahrung fundiert im senkrechten Weltbild [2], in dem die Welt mit all ihren Äusserungen in Entsprechungen gesehen wird. Man spricht in diesem Kontext auch vom analogen Denken, das das Leben nicht kausal, sondern gleichnishaft und in Ähnlichkeiten erfasst. Es werden also beispielsweise nicht alle Metalle oder alle Pflanzen zusammengefasst, sondern die Metalle bzw. Pflanzen werden verschiedenen Prinzipien zugeordnet, denen sie von ihrem Wesen und von ihrer Ausstrahlung her am nächsten sind. Die Naturheilkunde und Ganzheitsmedizin basieren (meist) auf dem analogen, systemischen Denken - im Gegensatz zu rationalen Wissenschaften, die sich dem linearen Denken verschrieben haben.

Im senkrechten Weltbild geben die sieben sichtbaren Planeten nicht nur zeitliche Rhythmen vor, sondern sind auch an der Ausgestaltung des Lebens mitbeteiligt. Diese Erfahrungen und Erkenntnisse sind eine wichtige Grundlage für verschiedenste Wissensgebiete der traditionellen Heilkunde. Babylonische, persische, arabische, jüdische und griechische Wurzeln werden hier sichtbar.

So hat die Sonne einen direkten Bezug zum menschlichen Herzen, zur Quelle der Lebensenergie, zu Her- zenswärme und Ausstrahlung, zu Sommer, Mittag und Sonntag. Mars wird beispielsweise sichtbar in Krankheiten wie Fieber und Entzündungen. In der Pflanzenwelt zeigt sich Merkur unter anderem in der Blattatmung und in feingliedrigen Pflanzen wie den Doldenblütlern [3]. Alle menschlichen Prozesse und die den Menschen umgebende Welt werden mit den sieben Planetenprinzipien verknüpft: Lebensalter, Temperamente, Charaktereigenschaften, Körperorgane, Körperfunktionen und Krankheitssymptome sowie die Wahl von Therapieverfahren und Heilmitteln usw. (Tab. 1).

Die Planeten werden auch in der Namensgebung der Wochentage erkennbar. Der Sonntag gilt der Sonne, der Montag dem Mond. Mars zeigt sich in der französischen Sprache in «Mardi» für Dienstag. Im französischen Wort «Mercredi» für Mittwoch klingt Merkur hindurch. Im Donnerstag erkennen wir «Donar», die nordische Variante von Jupiter, der sich im französischen Namen «Jeudi» für Donnerstag zeigt. Im Wort «Freitag» wird die Liebes- und Fruchtbarkeitsgöttin Freya bzw. Venus im französischen «Vendredi» genannt. Den Saturn erkennen wir im englischen Begriff «Saturday» für Samstag.

Tab. 1. Verknüpfung der sieben Metalle und ihre Bedeutung

\begin{tabular}{|c|c|c|c|c|}
\hline Metall & «Planet» & Gottheit (griech./röm.) & $\begin{array}{l}\text { Mythologische } \\
\text { Bedeutung }\end{array}$ & Beispiel einer Pflanze, die dem Metall «entspricht» \\
\hline Gold / Aurum (Au) & Sonne & Helios/Sol & Sonnengott & Hypericum perforatum (Johanniskraut) \\
\hline Quecksilber / Hydragyrum (Hg) & Merkur & Hermes/Merkur & Götterbote & Foeniculum vulgare (Fenchel) \\
\hline Kupfer / Cuprum $(\mathrm{Cu})$ & Venus & Aphrodite/Venus & Liebesgöttin & Rosa damascena (Rose) \\
\hline Eisen / Ferrum $(\mathrm{Fe})$ & Mars & Ares/Mars & Kriegsgott & Urtica dioica (Brennnessel) \\
\hline Zinn / Stannum (Sn) & Jupiter & Zeus/Jupiter & Göttervater & Taraxacum officinale (Löwenzahn) \\
\hline Blei / Plumbum (Pb) & Saturn & Kronos/Saturnus & Gott der Zeit & Equisetum arvense (Ackerschachtelhalm) \\
\hline
\end{tabular}

\section{KARGER}

Fax +497614520714 Information@Karger.com Information@Kar.
Chrischta Ganz

Praxis für Naturheilkunde

Steinentischstrasse 1, 8002 Zürich, Schweiz

naturheilpraxis@chrischtaganz.ch 


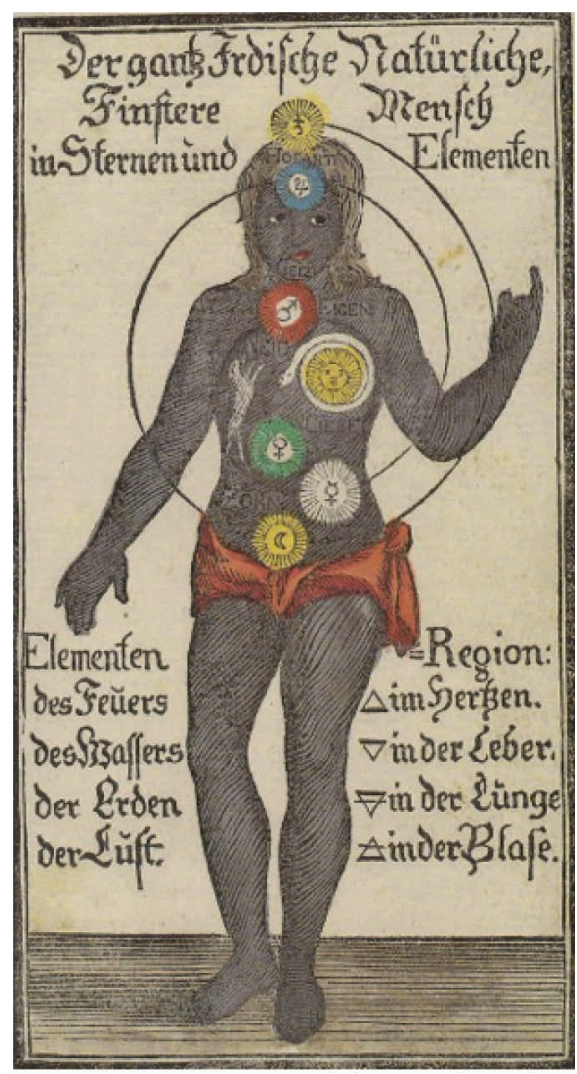

Abb. 1. Der Mensch und seine Organe in Verbindung mit den entsprechenden Elementen und Gestirnen (Georg Gichtl, 1638-1710, Theosophia practica).

\section{Die Metalle in der \\ Naturheilkunde}

Die sieben Planetenmetalle bereichern die Naturheilkunde auf ganz besondere Weise (Abb. 1), wie folgendes Zitat von Paracelsus aufzeigt: "Also versteht, dass das Hirn der Mond ist, die Lunge Mercurius, die Niere Venus, die Galle Mars, die Leber Jupiter, die Milz Saturn und das Herz ist die Sonne» [3].

Im Rahmen dieses Beitrags werden die Metalle im Überblick kurz vorgestellt.

\section{Gold (Aurum)}

Erst die Sonnenwärme ermöglicht das irdische Leben, weshalb ihre Kräfte als wohltätig und belebend für unser Dasein empfunden werden [4]. Dem Gold wird die Urkraft des Lebens, Vitalität und Aktivität, die Lebensenergie, das Licht, aber auch
Würde, Schönheit und Edelmut und die Herrschaft des Herzens zugeordnet, ebenso gutmütige Autorität sowie Ich-Bewusstsein, das Zentrum der Persönlichkeit und die Individualität [5].

Aurum wirkt im menschlichen Organismus auf die Blutzirkulation, das Herz-Kreislauf-System, die Augen (Sehkraft) sowie auf Gesamtbefindlichkeit, Vitalität und Stimmung.

\section{Silber (Argentum)}

Der Mond gilt als der Spiegel der Sonne und als sein Gegenpol - seine Wechselhaftigkeit ist ein deutlich spürbarer Wesenszug. Im Wechsel der Gezeiten oder im monatlichen Menstruationszyklus manifestieren sich seine Kräfte - der Mond bewirkt nicht nur Ebbe und Flut, sondern bewegt ebenso rhythmisch die Körperflüssigkeiten. So gilt Silber als Ausdruck der Seele, der Weiblichkeit, der Nacht, des Schlafes und der Regeneration. Zugeordnet werden dem Silber auch das Unterbewusstsein, die Phantasie, die Beweglichkeit, die Kraft des Wandels, die Wechselhaftigkeit und der Fluss der Lebenskraft [4].

Argentum wirkt im menschlichen Organismus auf die Körperflüssigkeiten, auf die weiblichen Organe, auf zyklische und hormonelle Prozesse wie beispielsweise die Monatsblutung, ebenso auf Schleimhäute, das Lymphsystem sowie auf Hypophyse, Hypothalamus und das limbische System.

\section{Quecksilber (Mercurius)}

Als einziges «bewegliches» Metall hat Quecksilber (Abb. 2) durch seinen speziellen Aggregatzustand eine Zwischenstellung inne. Es vermittelt zwischen Seele, Geist und Materie und

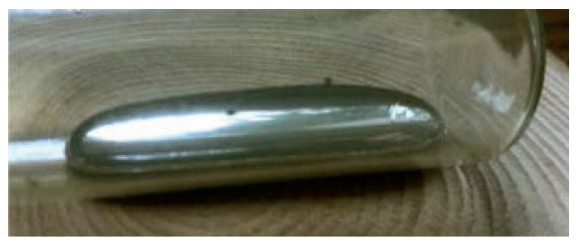

Abb. 2. Typische «Tropfenbildung» des Quecksilbers - Mercurius mit seiner Charakteristik, bei Raumtemperatur flüssig zu sein. steht für Kommunikation, Beweglichkeit, Intellekt, Logik, Sprache, Schrift sowie für Schlagfertigkeit und Forschergeist.

Mercurius wirkt im menschlichen Organismus auf die Atmungsorgane, die Sinnesorgane und die Nerven sowie auf alle Austauschprozesse (Atmung, Verdauung, Haut), das Hormonsystem, die Bewegung (Gliedmassen) und die Kommunikation (vergleiche auch Allergieneigung).

\section{Eisen (Ferrum)}

Eisen symbolisiert die Ich-Impulskraft. Das Körperliche steht über dem Geistigen und zeigt sich in Willensstärke, Tatenergie, Aktion, gezielter Bewegung, Entschlossenheit, Widerstandskraft, Aggression, Männlichkeit und Zeugungskraft.

Ferrum wirkt im menschlichen Organismus auf das Immunsystem (vergleiche auch Fieber und Entzündung), auf die Gallenflüssigkeit, auf Muskeln und Sehnen, auf die männlichen Organe sowie auf die Blutbildung, das Hämoglobin und die Sauerstoffverwertung, aber auch auf den Geruchssinn.

\section{Kupfer (Cuprum)}

Sanftheit, Schönheit, Harmonie, Anmut und Gleichgewicht, Liebe, Weiblichkeit wie auch Entspannung, Erweichung und Leichtigkeit werden dem Kupfer zugeordnet. Auch träumerische und schöpferische Qualitäten sowie Verführung und Bindung gehören zu Kupfer.

Cuprum wirkt im menschlichen Organismus auf die weiblichen Genitalien, auf Nieren, Prostata und Venen sowie auf das Fühlen und wird aufgrund seiner analgetischen, spasmolytischen und vegetativ ausgleichenden Wirkung vielseitig eingesetzt.

\section{Zinn (Stannum)}

Stannum ist ein weiches, biegsames Metall und steht für Formgebung, Entfaltungskraft, Fruchtbarkeit, Fülle, Ausdehnung, Erweiterung sowie 
für organisches Wachstum, Reife, Aufbau und Entwicklung, Grösse und Würde.

Stannum wirkt im menschlichen Organismus auf das Leber-Galle-System, die Ernährung, den Metabolismus (vergleiche Zivilisationskrankheiten) und den Geschmackssinn.

\section{Blei (Plumbum)}

Dem Blei werden Konzentration, Verhärtung, Verdichtung, Verlangsamung, Schwere, Ernst, Sicherheit, Alter, aber auch Konzentration, Beharrlichkeit sowie Melancholie, Argwohn und Geduld zugeordnet [3].

Plumbum wirkt im menschlichen Organismus auf die Milz, auf Knochen, Bänder, Zähne, Haut, Strukturbildung und alle Alterungsprozesse (vergleiche Chronifizierung eines Leidens) sowie auf den Gehörsinn.

\section{Bedeutung der Metalle in der heutigen Zeit}

Heute werden die Metalle als Heilmittel in einigen Therapierichtungen der Ganzheitsmedizin in diesem Sinne verwendet:

- Spagyrik: Die grundlegenden Wirkprinzipien der Metalle werden im spagyrischen Herstellungsprinzip für den Menschen ver-

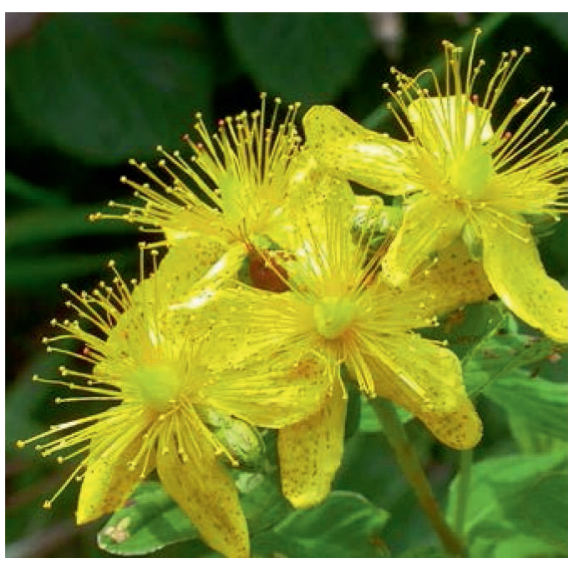

Abb. 3. Wird Hypericum mit Gold "gedüngt», so wird Gold nach anthroposophischer Sicht vegetabilisiert und für den Menschen «verfügbar» gemacht (Hypericum Auro cultum).

fügbar gemacht. Einige Hersteller von Spagyrika haben einen klar astromedizinischen Schwerpunkt.

- Anthroposophische Medizin: Viele astromedizinische Aspekte wurden von Rudolf Steiner aufgenommen und in die Anthroposophie integriert. So sind die sieben $\mathrm{Me}$ talle Gold, Silber, Eisen, Quecksilber, Kupfer, Zinn und Blei nicht aus dem anthroposophischen Heilmittelschatz wegzudenken. Die anthroposophische Medizin verwendet die Metalle nicht nur (in für den Menschen aufbereiteter Art) in Heilmitteln, die oral verabreicht werden, sondern kennt auch äusserliche Anwendungen wie Salben oder sogenannte Metallspiegelfolien. Eine wichtige Umsetzung ist auch die «Düngung» von bestimmten Heilpflanzen (Abb. 3) mit dem jeweiligen Metall zur Bereitstellung und zum Verfügbarmachen der jeweiligen Metallkraft: Dies wird als Vegetabilisierung eines Metalls bezeichnet.

- In der Homöopathie werden die Metalle anhand der Arzneimittelprüfungen eingesetzt.

- Auch die orthomolekulare Medizin verwendet Metalle (z.B. Ferrum). Im Rahmen dieses Artikels wird auf die stoffliche Wirkung der Metalle, wie sie die orthomolekulare Medizin verwendet, jedoch nicht eingegangen.

\section{Literatur}

1 Uecker DM: Metalle in der ganzheitlichen Therapie. Stuttgart, Sonntag, 2008.

2 Dahlke R, Klein N: Das senkrechte Weltbild. München, Hugendubel, 1986.

3 Raimann C, Ganz C, Garvelmann F, Bertschi-Stahl H-D, Fehr-Streule R: Grundlagen der Traditionellen Europäischen Naturheilkunde. Schiedlberg, Bacopa, 2012.

4 Madejsky M, Ochsner P, Amann M, Rippe O, Rätsch C: Paracelsusmedizin. Aarau, AT, 2004.

5 Madejsky M, Rippe O: Heilmittel der Sonne. Aarau/München, AT, 2013. 